\title{
Help Me in My Confusion: Should We Think More About Mammography and Colonoscopy as "Preference Sensitive Care'?
}

\author{
Joseph O’Donnell
}

Published online: 9 November 2010

(C) Springer 2010

I'm confused and I don't know how to resolve it and so I thought I'd put it out there for all of you to ponder.

This issue (and many of our prior issues) are loaded with articles about how to get more people (especially populations suffering from disparities) to be screened-with mammograms, colonoscopies, Pap smears or PSA's.

This approach stems from the viewpoint that it is key to diagnose cancers earlier or to remove/eradicate precancerous lesions. That is a worthy goal, but in practice, this territory is getting messier and hence my confusion.

I live in an institution (Dartmouth) that has the recent history of making trouble by raising questions and questioning assumptions. Our Godfather for these disruptions, Dr. Jack Wennberg (I'd highly recommend his autobiographical book called Tracking Medicine; A Researcher's Quest to UnderStand Healthcare [1]) started the whole field that he has coined "the evaluative clinical sciences". Jack noticed (isn't it great that a first step often occurs when one notices and wonders about something) that in his own town in Vermont, all of his children's friends had had their tonsils out, whereas in nearby towns this wasn't the case. He began looking at variations for other things like Cesarean Sections and they varied widely too. He became fascinated by these observations and has devoted his career to trying to find out why such variations occur. The answer it has turned out is not because of applying the right science to these problems. These variations are due to many other things. We call these practice patterns.

When Mrs. Clinton was designing the plan she was trying to implement to bring about healthcare reform, she

J. O’Donnell $(\bowtie)$

Dartmouth Medical School,

Hanover, NH 03755, USA

e-mail: joseph.f.odonnell@dartmouth.edu really resonated with Dr. Wennberg's findings and thought dealing with variations might be one way to control costs and get higher quality of care. (Value is directly related to quality and inversely related to cost.) As we all know, her plan was crushed, but Jack and his colleagues who had produced reams of data decided to use it by publishing something called the Dartmouth Atlas of Healthcare which details the profound variations that characterize our healthcare system. He thought that studying these data could yield insights.

Jack and his colleagues in subsequent work have divided care into what they call effective care, supply sensitive care and preference sensitive care. Effective care includes things like getting a hip replacement after a fractured hip, or getting beta blockers after an MI or in our field, getting Pap smears. For supply sensitive care, there is more care where there are conditions like more beds, more specialists, and more technology; for instance, where there are more cardiologists, patients are seen more frequently, costs rise but outcomes do not differ. There were several presentations at this year's annual meeting, particularly those by editorial board member, Dr Frank Johnson, that address issues of supply sensitive care. I will ask him to write an editorial on this for a future issue.

The third type of care, preference sensitive care, is where I'm confused. A preference sensitive decision might be when or whether to do a knee replacement for someone with degenerative joint disease in the knee. It is very clear to me now that the use of the PSA lies within this preference sensitive domain. It depends on values, risks and benefits and is not just a simple decision. The strategy of shared decision making is the way to approach a decision like getting a PSA and that is how we are applying this decision-or at least should be applying or teaching how to apply! 
Mammography is a strategy that is looking more to me like a preference sensitive decision, particularly in the decade of the forties. There is no question that breast cancer is a far too common and far too lethal disease, but not in everyone. One of my colleagues at Dartmouth, Dr Gil Welch, has convinced me that there are cases that the woman will die with it rather than of it. And, there is no question that mammography saves lives. The questions appear to me to be about risks and benefits and ultimately, values. Mammography can lead to worry, biopsies, costs and other things. It is not a slam dunk to say that a solution to disparities is just to have women get mammograms, which is what many articles in this journal seem to imply. I suspect like PSA, we need to teach about shared decision making.

Consider also these things about colon cancer screening. There is no doubt that this works to prevent deaths too, but here are some statistics to ponder: 6 of 100 people at average risk will get colon cancer in their lifetime; 3 of those 6 will die if they never get screened; $1-2$ will die even if screened-so 1-2 fewer people of 100 will die if they get screened. Colonoscopy every 10 years is the most effective screen; polyps are found in 20/100 patients, perforation happens in $1 / 1000$, bleeding requiring hospitalization post-polyp removal in $1 / 100$ and death in $1 / 25000$ 50000. Stool cards have also been shown to be an effective test and 10/100 are positive and then require colonoscopy. Barium enemas are abnormal in 20/100, likely resulting in colonoscopy. Consider the costs and discomfort of these. Should the decision about which test be a preference sensitive, shared decision? Do we have enough discussion about costs, risks, inconvenience? What do we teach our students? What is right to teach?

In addition to what we teach, we have other pulls. In my practice, there are quality measures for colonoscopy, and mammography that make them seem to me like they are not preference sensitive. They are on our "tests" to see if we are considered a good, effective physician or healthcare system.

As I consider this business of preference sensitive care, I really am perplexed. In the second year medical school oncology course I taught, I used to have my students memorize the ACS screening guidelines and I'd design test questions to ask about these, but I now realize I was being too naïve about a complex situation. And also, by putting so much focus on these things and too much hope in early diagnosis, I didn't fully appreciate things that surprised me like the role of postmenopausal hormone replacement in the etiology of breast cancer. I don't think I truly understand the role of exercise, weight control, diet and poverty in disparities but if we just focus on "getting the testy", we may miss other opportunities.
I want breast cancer and colon cancer eliminated. Here's a few more thoughts as I mature as an oncologist as I struggle to teach about the use of mammography and place of colonoscopy.

I believe we ought to put tons of emphasis on defining the characteristics of the "bad actor" cancers that kill people. We ought to diagnose these early and hammer them with effective therapy. Another thought is that we ought to create really useful decision aids that allow patients to understand the risks and benefits more clearly and make decisions using their values. I think we could have the data to put on a hand held device that could show the particular woman in front of us, her risks of developing breast cancer in the next 10,20, 30 years, how mammography could lower these, or expose her to other risks. Then, she and I as her provider, could make a value based shared decision. The same could be done with the choice of screening for colorectal cancer. For breast cancer, I think there ought to be a convening of a panel of women, not just the so called experts but people who are real experts on their bodies, to present them the controversies and help us all to define a consensus about mammography that incorporates values and preferences. We might even try to figure out how Sweden does this so much better and at less cost [2]. Something like this would take the consumer's perspectives into sharp focus, and may help lead me out of my confusion, and us out of our simple strategy of getting more tests to eliminate breast and colon cancer. Meanwhile, we ought to keep our minds open and look for surprises, like the postmenopausal estrogen story, all the while keeping focus on physical activity, weight control, healthy diet, environmental toxins and the big kahuna, poverty.

I remember once asking Partners in Health founder Paul Farmer what one thing he would do to elimate HIV/AIDS in some of the poor countries like Haiti where he worked. He surprised me with his answer which was; "get women jobs!" But that makes sense, doesn't it?

What do you think? Help me in my confusion. What can we do?

\section{References}

1. Wennberg J (2010) Tracking medicine: a researcher's quest to understand healthcare. Oxford University Press, New York

2. Hellquist BN, Duffy SW, Abdsaleh S, Bjorneld L, Bordas P, Tabar L, Vitak B, Zackrisson S, Nystrom L, Jonsson H (2010) Effectiveness of population-based service screening with mammography for women ages 40 to 49 years: evaluation of the Swedish Mammography Screening in Young Women (SCRY) Cohort. Cancer. doi:10.1002/cncr.25650 